\title{
Mass Spectrometric Identification of Oxidative Modifications of Tryptophan Residues in Proteins: Chemical Artifact or Post-Translational Modification?
}

\author{
Irina Perdivara, ${ }^{\mathrm{a}, \mathrm{b}}$ Leesa J. Deterding, ${ }^{\mathrm{a}}$ Michael Przybylski, ${ }^{\mathrm{b}}$ and \\ Kenneth B. Tomer ${ }^{a}$ \\ ${ }^{a}$ Laboratory of Structural Biology, National Institute of Environmental Health Sciences, Research Triangle \\ Park, Research Triangle Park, North Carolina, USA \\ ${ }^{b}$ Laboratory of Analytical Chemistry and Biopolymer Structure Analysis, University of Konstanz, Konstanz, \\ Germany
}

Oxidative modification of tryptophan to kynurenine (KYN) and N-formyl kynurenine (NFK) has been described in mitochondrial proteins associated with redox metabolism, and in human cataract lenses. To a large extent, however, previously reported identifications of these modifications were performed using peptide mass fingerprinting and/or tandem-MS data of proteins separated by gel electrophoresis. To date, it is uncertain whether NFK and KYN may represent sample handling artifacts or exclusively post-translational events. To address the problem of the origin of tryptophan oxidation, we characterized several antibodies by liquid chromatography-tandem mass spectrometry, with and without the use of electrophoretic separation of heavy and light chains. Antibodies are not normally expected to undergo oxidative modifications, however, several tryptophan (Trp) residues on both heavy and light chains were found extensively modified to both doubly oxidized Trp and KYN following SDS-PAGE separation and in-gel digestion. In contrast, those residues were observed as non-modified upon in-solution digestion. These results indicate that Trp oxidation may occur as an artifact in proteins separated by SDS-PAGE, and their presence should be carefully interpreted, especially when gel electrophoretic separation methods are employed. (J Am Soc Mass Spectrom 2010, 21, 1114-1117) (C) 2010 American Society for Mass Spectrometry

$I^{\prime}$ n vivo, tryptophan (Trp) residues may undergo extensive oxidative modification upon exposure to UV light and oxidative agents [1-4]. The structures of oxidatively modified Trp residues are summarized in Figure S-1, (Supplemental Information, which can be found in the electronic version of this article). Peptides bearing oxidized Trp modifications generally exhibit mass increases of 4,16 , and $32 \mathrm{Da}$, corresponding to the formation of kynurenine (KYN), hydroxytryptophan $\left(\mathrm{W}_{\mathrm{ox} 1}\right)$, and $\mathrm{N}$-formylkynurenine/dihydroxytryptophan (NFK/ $\mathrm{W}_{\mathrm{ox} 2}$, referred to also as "doubly oxidized Trp"), and their combinations, such as hydroxykynurenine $\left(\mathrm{KYN}_{\mathrm{ox} 1},+20 \mathrm{Da}\right)$. Oxidation to hydroxytryptophan $\left(\mathrm{W}_{\mathrm{ox} 1}\right)$ has been observed as a result of sample handling, e.g.; following protein separation by gel electrophoresis [5]. Trp modification to NFK and KYN and degradation have been described in mitochondrial proteins associated with redox metabolism $[6,7]$ in human

Address reprint requests to Dr. K. B. Tomer, Laboratory of Structural Biology, National Institutes of Environmental Health Sciences, 111 T. W. Alexander Drive, MD F0-03, Research Triangle Park, NC 27709, USA. E-mail: tomer@niehs.nih.gov and Dr. M. Przybylski, Department of Chemistry, University of Konstanz, Universitätsstrasse 10, 78457 Konstanz, Germany. E-mail: Michael.Przybylski@uni-konstanz.de cataract lenses [8, 9], and upon photolytic oxidation [10]. Modified proteins have been proposed as markers of oxidative stress, e.g.; in atherosclerosis [11]. Some authors have suggested ion abundances of modified Trp, $W_{\text {ox } 1}$, and NFK/ $W_{\text {ox } 2}$ peptides should be included in protein database search algorithms to improve the identification score [12].

Based on the literature, it is uncertain whether oxidation products such as NFK and KYN identified upon electrophoresis represent artifacts upon sample isolation and purification [5, 13, 14], or true post-translational modifications. A number of previous proteomic studies have reported the identification of oxidative modifications of Trp using peptide mass fingerprinting of proteins separated by gel electrophoresis [6, 7, 15-17]. To address the problem of the potential artifactual nature of Trp oxidation, we have used liquid chromatography tandem mass spectrometry (LC/MS/MS), with and without gel electrophoretic separation, to characterize a monoclonal antibody (MAb), which is a secreted glycoprotein normally not expected to undergo oxidative modifications [18]. Our results indicate Trp oxidative modifications to (Trp $+32 \mathrm{Da})$ and KYN occur as artifacts in proteins separated by sodium dodecyl sul- 
fate polyacrylamide gel electrophoresis (SDS-PAGE). Hence, care should be taken in the interpretation of data suggesting a correlation between tryptophan oxidation and oxidative stress in vivo.

\section{Materials and Methods}

Mouse anti-human $\beta$-amyloid precursor protein $\mathrm{MAb}$ (clone 6A6) was purchased from US Biological (Swampscott, MA, USA). Dithiothreitol, iodoacetamide, ammonium bicarbonate, and 96\% formic acid were purchased from Sigma-Aldrich (St. Louis, MO, USA). Sequencing grade-modified porcine trypsin was obtained from Promega (Madison, WI, USA) and sequencing grade bovine $\alpha$-chymotrypsin was obtained from Roche (Penzberg, Germany). NuPage $4 \%-12 \%$ Bis-Tris precast gels, sample and running buffers, and Coomassie SimplyBlue were purchased from Invitrogen (Carlsbad, CA, USA). Acetonitrile was purchased from Caledon Laboratories, Ltd. (Georgetown, ON, Canada). Purified water $(17.8 \mathrm{M} \Omega$ ) was obtained from an in-house Hydro Picopure 2 system.

\section{Sample Preparation, SDS-PAGE and In-Gel Digestion}

Samples were reduced and alkylated before electrophoresis as follows: monoclonal antibody 6A6 stock solution $(20 \mu \mathrm{L} ; 0.5 \mu \mathrm{g} / \mu \mathrm{L}$ in PBS buffer) was incubated for $1 \mathrm{~h}$ at $95^{\circ} \mathrm{C}$ with $20 \mu \mathrm{L}$ sample loading buffer containing $100 \mathrm{mM}$ dithiothreitol, and iodoacetamide in water was added at a molar ratio DTT/IAA of 1:3. The reaction was continued for $1 \mathrm{~h}$ at room temperature. The reduced and alkylated antibody was loaded onto the gel and the heavy and light chains were separated. Electrophoresis was performed at $200 \mathrm{~V}$ and a maximum of $80 \mathrm{~mA}$ for $1 \mathrm{~h}$. The bands were stained with Coomassie SimplyBlue solution. The protein bands corresponding to antibody heavy and light chains were excised and digested with trypsin or with chymotrypsin for $8 \mathrm{~h}$ at $37^{\circ} \mathrm{C}$ in an automated fashion with a Progest robotic digester (Genomic Solutions, now part of Digilabs, Holliston, MA, USA). In-gel digestion with $\alpha$-chymotrypsin was performed manually using an enzyme to substrate ration of 1:30, based on the initial amount of reduced and alkylated antibody. Samples were lyophilized to dryness and resuspended in $0.1 \%$ formic acid.

\section{In-Solution Digestion}

MAb samples were reduced as above except that a 100 $\mathrm{mM}$ aqueous DTT solution was used. Alkylation was performed as above. Following alkylation for $1 \mathrm{~h}$ at room temperature, the reaction mixture was divided in two aliquots $\times 20 \mu \mathrm{L}$, of which one was digested with trypsin and one with chymotrypsin. To each vial, $40 \mu \mathrm{L}$ of a $25 \mathrm{mM}$ solution of trypsin or chymotrypsin at an enzyme:substrate ratio of $1: 50$ and sufficient ammonium carbonate to maintain a $\mathrm{pH}$ of 7.4 was added to each vial and the digestions were performed over night at $37^{\circ} \mathrm{C}$.

\section{Mass Spectrometry and Database Search}

LC/MS analyses were performed on a Waters Q-TOF Premier mass spectrometer (Waters, Milford, MA, USA) and the MS data were searched using Mascot software (Matrix Science, Manchester, UK) as previously described [19].

\section{Results and Discussion}

\section{MS Identification of Oxidative Trp Modifications}

To determine the nature and extent of tryptophan oxidation derived from sample handling procedures,

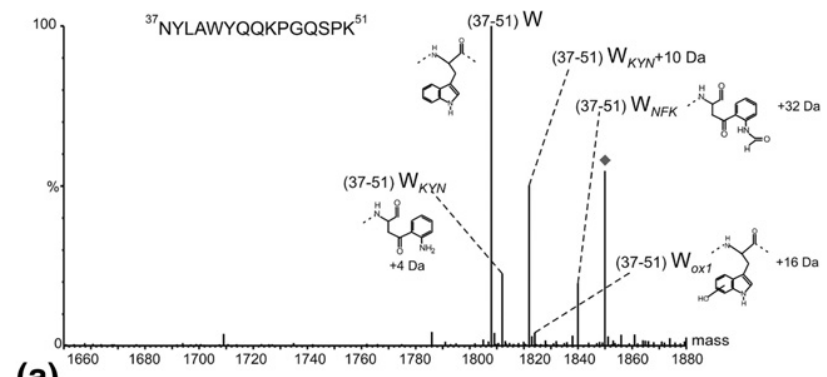

(a)
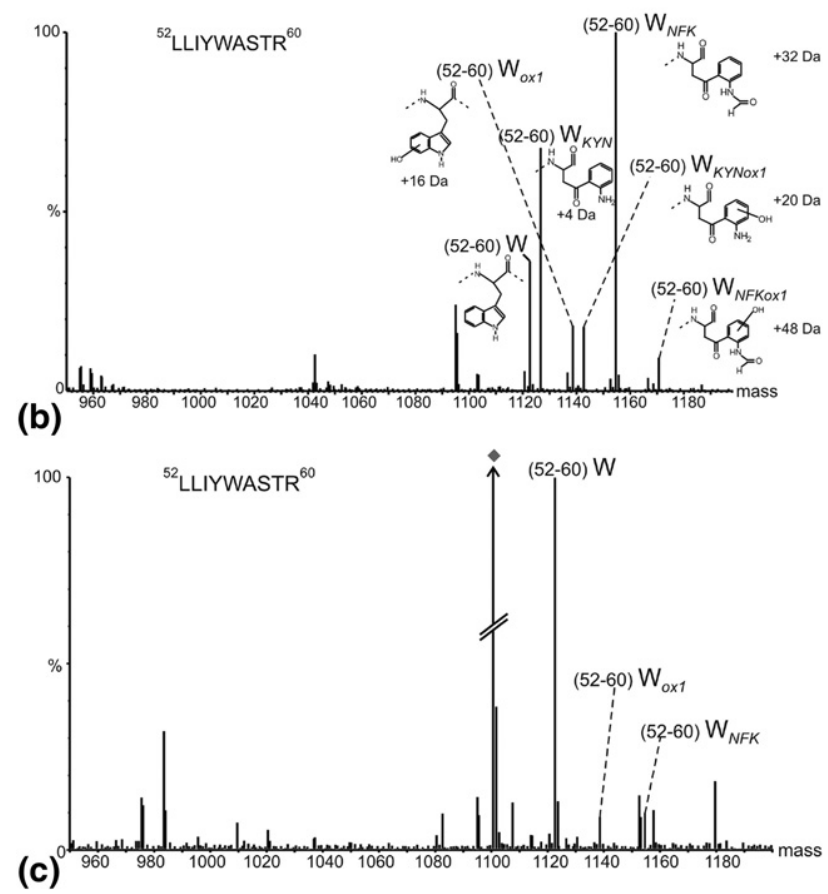

Figure 1. Deconvoluted mass spectra showing the heterogeneity and the relative abundance of peptides containing Trp and its oxidative modifications: (a) light chain peptide (37-51), after in-gel digestion (diamond indicates a nonrelated ion); (b) light chain peptide (52-60), after in-gel digestion; (c) light chain peptide (52-60) after digestion in-solution (diamond in (c) indicates a nonrelated, heavy chain peptide ion). 
the amino acid sequence of a MAb 6A6 was analyzed using an LC-MS/MS approach, which employed reduction, alkylation, and proteolytic degradation of the antibody (1) in-solution, and (2) following SDS-PAGE separation of the heavy and light chains. Both experiments were performed under identical experimental conditions with regard to reduction and alkylation, as described in the experimental section.

$\mathrm{MAb} 6 \mathrm{~A} 6$ contains four Trp residues in the light chain and eight in the heavy chain. Following in-solution digestion with trypsin, LC-MS/MS and NCBInr database search [19], these residues were identified as unmodified, suggesting that this MAb is not primarily oxidized during storage, as previously reported for Trp residues in an MAb [18]. Upon SDS-PAGE separation, pronounced molecular heterogeneity due to various oxidative modifications of the majority of Trp residues was observed. Peptides bearing oxidative Trp modifications exhibited characteristic mass shifts of $+4 \mathrm{Da}(\mathrm{KYN})$, +16 Da (singly oxidized Trp), +32 Da $\left(\mathrm{NFK} / \mathrm{W}_{\text {ox2 }}\right.$, "doubly oxidized Trp"), and even +48 Da (attributable to hydroxy-NFK, $\left.\mathrm{NFK}_{\mathrm{ox} 1}\right)$. In the case of (Trp +32 Da), the modifications may represent either NFK or dihydroxy$\operatorname{Trp}\left(\mathrm{W}_{\text {ox2 }}\right)$ [20]. Because these isobaric structures were identified solely by MS, the authors refrain from making structural assignments to this mass. Examples showing the distribution of oxidation products in the tryptic peptides (37-51) and (52-60) of the MAb 6A6 light chain, are presented in Figure $1 \mathrm{a}$ and $\mathrm{b}$. The most abundant ions in Figure 1a $(\mathrm{m} / \mathrm{z}$ 1807.911) were assigned to the unmodified peptide (37-51) (calculated $\mathrm{m} / \mathrm{z}$ 1807.913) followed by the same peptide containing both KYN (observed $m / z$ 1811.922, calculated $m / z, 1811.908$ ), and an additional unidentified modification (+10 Da) (observed $m / z$ 1821.897, Figure S-2), and by the (Trp +32 Da)-modified peptide (observed $m / z$ 1839.921, calculated $m / z$ 1839.903). The most abundant Trp modification observed for peptide (52-60) (Figure 1b) was assigned to the "double Trp oxidation" (+32 Da) (observed $m / z$ 1154.638, calculated $m / z$ 1154.630), while the unmodified peptide (observed $\mathrm{m} / \mathrm{z}$ 1122.622, calculated $m / z$ 1122.631) was observed with lower relative abundance. The MS/MS spectra of these peptides were manually interrogated (see Fig. S-3), and generally revealed that the oxidative Trp modifications did not create signature fragment ions, as previously noted [21]. An additional example of a heavy chain tryptic peptide, non-modified, and simultaneously containing one KYN and one Trp +32Da modifications, is shown in Fig. S-4. The oxidized Trp peptides were generally found to elute earlier than the unmodified peptides (Fig. S-5).

Tryptic peptide (52-60), however, showed minimal oxidation when LC-MS/MS analysis of an in-solution digest was performed (Figure 1c); these products were presumably formed during electrospray ionization [22] because the chromatographic retention times of oxidized and non-oxidized species were identical. Reported differences in the retention times of peptides containing oxidized Trp versus unmodified Trp can be used to discriminate between electrospray induced oxidation [22] and those derived from oxidation before electrospray.

Table 1 summarizes the observed Trp-containing peptides, their oxidative modifications, and the ion abundances of the oxidized products relative to the unmodified peptides from both solution and in-gel digests. The most frequently observed modifications were $\mathrm{KYN}, \mathrm{W}_{\mathrm{ox} 1}$, and $(\operatorname{Trp}+32 \mathrm{Da})$, whereas $\mathrm{KYN}_{\mathrm{ox} 1}(+20 \mathrm{Da})$ and $\mathrm{NFK}_{\mathrm{ox} 1}(+48$ $\mathrm{Da})$ products were found with lower relative abundance. However, no trend in the relative abundances of these oxidation products in different peptides could be determined, suggesting that the amino acids in the vicinity of Trp may be of crucial importance for the formation of a specific product. From our data, Trp oxidation appears to be dependent on the amino acid microenvironment around a Trp residue. However, a more detailed study is needed to evaluate possible sequence specificities of Trp oxidation in peptides.

The source of the reactive oxygen species leading to the pattern of Trp oxidation is yet unclear. The high

Table 1. Antibody tryptic and chymotryptic Trp-containing peptides and their oxidative modifications observed by LC-MS/MS

\begin{tabular}{|c|c|c|c|c|c|c|c|c|c|c|c|c|}
\hline \multirow[b]{3}{*}{ Peptide sequence } & \multicolumn{12}{|c|}{ Tryptophan modifications/relative abundance* } \\
\hline & \multicolumn{2}{|c|}{ None } & \multicolumn{2}{|c|}{ KYN } & \multicolumn{2}{|c|}{ Wox1 } & \multicolumn{2}{|c|}{ KYNox1 } & \multicolumn{2}{|c|}{ NFK } & \multicolumn{2}{|c|}{ NFKox1 } \\
\hline & Gel & Sol & Gel & Sol & Gel & Sol & Gel & Sol & Gel & Sol & Gel & Sol \\
\hline LLIYWASTR & 1 & 1 & 1.85 & - & 0.5 & 0.06 & 0.5 & - & 2.7 & 0.07 & 0.25 & - \\
\hline NYLAWYQQKPGQSPK & 1 & 1 & 0.21 & - & 0.04 & - & - & - & 0.17 & 0.01 & - & - \\
\hline QNGVLNSWTTDODSK & 1 & 1 & 0.37 & - & 0.73 & 0.01 & 0.03 & - & 0.51 & 0.01 & 0.05 & - \\
\hline WKIDGSER & 1 & 1 & 0.32 & - & - & - & - & - & 0.32 & - & - & - \\
\hline SNWEAGNTFTC(alk)SVLHEGLHNHHTEK & 1 & 1 & 0.05 & - & 0.75 & 0.03 & - & - & 0.35 & 0.03 & 0.15 & 0.01 \\
\hline SVSELPIM(ox)HODWLNGK & 1 & 1 & 0.14 & - & 0.43 & - & 0.03 & - & 0.22 & & 0.11 & - \\
\hline GNYVGPM(ox)DYWGQGTSVTVSSAK & 1 & 1 & 0.13 & - & 0.34 & 0.02 & - & - & 0.14 & 0.02 & - & - \\
\hline GC(alk)LVKGYFPEPVTVT $\underline{W}$ & 1 & 1 & - & - & 0.61 & 0.04 & - & - & 0.16 & 0.05 & 0.08 & - \\
\hline SWFVDDVEVH & 1 & 1 & 0.03 & - & 0.05 & 0.02 & - & - & 0.06 & 0.06 & - & - \\
\hline
\end{tabular}

* The relative abundance of each (oxidized) Trp containing species was determined relative to the ion abundance of the unmodified peptide. For each peptide, the calculations were performed using the mass spectrum, obtained by averaging the mass scans over the chromatographic retention time window in which oxidized and non-oxidized peptides elute. All charge states observed for a peptide species were considered in the determination of their relative abundances. 
voltage employed for SDS-PAGE may cause the formation of small amounts of ozone in the electrophoretic cell, which in turn may initiate a reaction cascade leading to Trp oxidation products. This hypothesis is supported by a previous study showing that ozone in ambient air can cause oxidation [23].

\section{Conclusion}

We have shown that oxidative modifications of Trpcontaining peptides to (Trp $+32 \mathrm{Da})$ and KYN can represent artefacts derived from sample preparation using gel electrophoresis. The extent of oxidation and product distribution in a specific Trp-containing peptide appears to depend on the amino acid microenvironment around the Trp residue. These results are important in the context of proteomics studies using SDS-PAGE aimed at the identification of biological oxidative modifications in proteins, as different sample preparation techniques may introduce unexpected modifications, which may lead to over-interpretation of data.

\section{Acknowledgments}

The authors acknowledge support for this work, in part, by the Intramural Research Program of the National Institutes of Environmental Health Sciences/National Institutes of Health (project ES050171), and by the Deutsche Forschungsgemeinschaft, Bonn, Germany (PR-175-13-1 and FO-753).

\section{Appendix A Supplementary Material}

Supplementary material associated with this article may be found in the online version at doi:10.1016/ j.jasms.2010.02.016.

\section{References}

1. Alvarez, B.; Radi, R. Peroxynitrite Reactivity with Amino Acids and Proteins. Amino Acids 2003, 25(3-4), 295-311.

2. Davies, M. J.; Truscott, R. J. Photo-Oxidation of Proteins and Its Role in Cataractogenesis. J. Photochem. Photobiol. B 2001, 63(1-3), 114-125.

3. Takamoto, K.; Chance, M. R. Radiolytic Protein Footprinting with Mass Spectrometry to Probe the Structure of Macromolecular complexes. Annu. Rev. Biophys. Biomol. Struct. 2006, 35, 251-276.

4. Bregere, C.; Rebrin, I.; Sohal, R. S. Detection and Characterization of In Vivo Nitration and Oxidation of Tryptophan rResidues in Proteins. Methods Enzymol. 2008, 441, 339-349.
5. Froelich, J. M.; Reid, G. E. The Origin and Control of Ex Vivo Oxidative Peptide Modifications Prior to Mass Spectrometry Analysis. Proteomics 2008, 8(7), 1334-1345.

6. Taylor, S. W.; Fahy, E.; Murray, J.; Capaldi, R. A.; Ghosh, S. S. Oxidative Post-Translational Modification of Tryptophan Residues in Cardiac Mitochondrial Proteins. J. Biol. Chem. 2003, 278(22), 19587-19590.

7. Moller, I. M.; Kristensen, B. K. Protein Oxidation in Plant Mitochondria Detected as Oxidized Tryptophan. Free Radic. Biol. Med. 2006, 40(3), $430-435$.

8. Hains, P. G.; Truscott, R. J.; Post-Translational Modifications in the Nuclear Region of Young, Aged, and Cataract Human Lenses. J. Proteome Res. 2007, 6(10), 3935-3943.

9. MacCoss, M. J.; McDonald, W. H.; Saraf, A.; Sadygov, R.; Clark, J. M.; Tasto, J. J.; Gould, K. L.; Wolters, D.; Washburn, M.; Weiss, A.; Clark, J. I.; Yates, J. R. III. Shotgun Identification of Protein Modifications from Protein Complexes and Lens Tissue. Proc. Natl. Acad. Sci. U.S.A. 2002, 99(12), 7900-7905.

10. Kunz, L.; Zeidler, U.; Haegele, K.; Przybylski, M.; Stark, G. Photodynamic and Radiolytic Inactivation of Ion Channels Formed by Gramicidin A: Oxidation and Fragmentation. Biochemistry 1995, 34(37), 1189511903.

11. Obama, T.; Kato, R.; Masuda, Y.; Takahashi, K.; Aiuchi, T.; Itabe, H. Analysis of Modified Apolipoprotein B-100 Structures Formed in Oxidized Low-Density Lipoprotein Using LC-MS/MS. Proteomics 2007, 7(13), 2132-2141.

12. Thiede, B.; Lamer, S.; Mattow, J.; Siejak, F.; Dimmler, C.; Rudel, T. Jungblut, P. R. Analysis of Missed Cleavage Sites, Tryptophan Oxidation, and N-Terminal Pyroglutamylation After In-Gel Tryptic Digestion. Rapid Commun. Mass Spectrom. 2000, 14(6), 496-502.

13. Staniszewska, M.; Nagaraj, R. H.; Detection of Kynurenine Modifications in Proteins Using a Monoclonal Antibody. I. Immunol. Methods 2007, 324(1-2), 63-73.

14. Zhang, X.; Shi, L.; Shu, S.; Wang, Y.; Zhao, K.; Xu, N.; Liu, S.; Roepstorff, P. An Improved Method of Sample Preparation on AnchorChip Targets for MALDI-MS and MS/MS and Its Application in the Liver Proteome Project. Proteomics 2007, 7(14), 2340-2349.

15. Bienvenut, W. V.; Deon, C.; Pasquarello, C.; Campbell, J. M.; Sanchez, J. C.; Vestal, M. L.; Hochstrasser, D. F. Matrix-Assisted Laser Desorption/ Ionization-Tandem Mass Spectrometry with High Resolution and Sensitivity for Identification and Characterization of Proteins. Proteomics 2002, 2(7), 868-876

16. Karty, J. A.; Ireland, M. M.; Brun, Y. V.; Reilly, J. P. Artifacts and Unassigned Masses Encountered in Peptide Mass Mapping. J. Chromatogr. B Analyt. Technol. Biomed. Life Sci. 2002, 782(1-2), 363-383.

17. Lemma-Gray, P.; Weintraub, S. T.; Carroll, C. A.; Musatov, A.; Robinson, N. C. Tryptophan 334 Oxidation in Bovine Cytochrome $c$ Oxidase Subunit I Involves Free Radical Migration. FEBS Lett. 2007, 581(3) 437-442.

18. Yang, J.; Wang, S.; Liu, J.; Raghani, A. Determination of Tryptophan Oxidation of Monoclonal Antibody by Reversed Phase High Performance Liquid Chromatography. J. Chromatogr. A 2007, 1156(1-2), 174182.

19. Perdivara, I.; Deterding, L.; Moise, A.; Tomer, K. B.; Przybylski, M. Determination of Primary Structure and Microheterogeneity of a $\beta$-Amyloid Plaque-Specific Antibody Using High-Performance LCTandem Mass Spectrometry. Anal. Bioanal. Chem. 2008, 391(1), 325-336.

20. Yamakura, F.; Matsumoto, T.; Ikeda, K.; Taka, H.; Fujimura, T.; Murayama, K.; Watanabe, E.; Tamaki, M.; Imai, T.; Takamori, K. Nitrated and Oxidized Products of a Single Tryptophan Residue in Human $\mathrm{Cu}$, Zn-Superoxide Dismutase Treated with Either Peroxynitrite-Carbon Dioxide or Myeloperoxidase-Hydrogen Peroxide-Nitrite. J. Biochem. 2005, 138(1), 57-69.

21. Swiderek, K. M.; Davis, M. T.; Lee, T. D. The Identification of Peptide Modifications Derived from Gel-Separated Proteins Using Electrospray Triple Quadrupole and Ion Trap Analyses. Electrophoresis 1998, 19(6), 989-997.

22. Chen, M.; Cook, K. D. Oxidation Artifacts in the Electrospray Mass Spectrometry of A- $\beta$ Peptide. Anal. Chem. 2007, 79(5), 2031-2036.

23. Cohen, S. L. Ozone in Ambient Air as a Source of Adventitious Oxidation. A Mass Spectrometric Study. Anal. Chem. 2006, 78(13), 4352-4362. 